\title{
Numerical simulation and experimental verification on downwash air flow of six-rotor agricultural unmanned aerial vehicle in hover
}

\author{
Yang Fengbo, Xue Xinyu*, Zhang Ling, Sun Zhu \\ (Nanjing Research Institute for Agricultural Mechanization, Ministry of Agriculture, Nanjing 210014, China)
}

\begin{abstract}
Recently, multi-rotor unmanned aerial vehicle (UAV) becomes more and more significantly irreplaceable in the field of plant protection against diseases, pests and weeds of crops. The easy takeoff and landing performance, hover function and high spraying efficiency of UAV are urgently required to spray pesticide for crop timely and effectively, especially in dispersed plots and hilly mountains. In such situations, the current researches about UAV spray application mainly focus on studying the influence of the UAV spraying parameters on the droplet deposition, such as operation height, operation velocity and wind velocity. The deposition and distribution of pesticide droplets on crops which depends on installation position of nozzle and airflow distribution characteristics of UAV are directly related to the control effect of pesticide and crop growth in different growth periods. As a preliminary step, this study focuses on the dynamic development law and distribution characteristics of the downwash air flow for the SLK-5 six-rotor agricultural UAV. Based on compressible Reynolds-averaged Navier-Stokes (RANS) equations with an RNG $k-\varepsilon$ turbulence model and dynamic mesh technology, the efficient three-dimensional computational fluid dynamics (CFD) method was established to analyze the flow field distribution characteristics of UAV in hover. Then the unsteady interaction flow field of the wing was investigated in detail. The downwash wind speed of the marked points for the SLK-5 UAV in hover was also tested by weather tracker. It was found that the maximum velocity value of the downwash flow was close to $10 \mathrm{~m} / \mathrm{s}$; the $z$-direction velocity was the main body of the wind velocity in the downwash airflow, and the comparison of the wind velocity experiment test and simulation showed that the relative error was less than $12 \%$ between the experimental and simulated values of the $z$-direction velocity at the marked points. Then the flow characteristics of the longitudinal and cross section were analyzed in detail, the results obtained can be used as a reference for drift and sedimentation studies for multi-rotor unmanned aerial vehicle.
\end{abstract}

Keywords: UAV, downwash air flow, numerical simulation, experimental verification, pesticide spray, wing interference DOI: $10.25165 /$ j.ijabe.20171004.3077

Citation: Yang F B, Xue X Y, Zhang L, Sun Z. Numerical simulation and experimental verification on downwash air flow of six-rotor agricultural unmanned aerial vehicle in hover. Int J Agric \& Biol Eng, 2017; 10(4): 41-53.

\section{Introduction}

Aerial spray application which has a history of more than a century is one of the major forms of pesticide

Received date: 2016-12-03 Accepted date: 2017-04-20

Biographies: Yang Fengbo, PhD, Post Doctor, Assistant Research Fellow, research interests: precise pesticide spraying, Email: yangfengbo.cool@163.com; Zhang Ling, Professor, research interests: crop protection and machinery engineering, Email: nnnz11965@126.com; Sun Zhu, Master, Assistant Research Fellow, research interests: crop protection and machinery engineering, Email: 43148588@qq.com.

* Corresponding author: Xue Xinyu, PhD, Professor, research interests: crop protection and machinery engineering. Nanjing Research Institute for Agricultural Mechanization, Nanjing 210014, China. Tel: +86-84346243, Email: 735178312@qq.com. application $^{[1]}$, and the fixed wing aircraft is the major application carrier typically. However, in recent years, unmanned aircraft systems (UASs), also referred to as unmanned aerial vehicles (UAVs), have been applied in many fields and captured the public attentions ${ }^{[2,3]}$. The agricultural UAV offers potential significant contributions to agriculture ${ }^{[4]}$, it is the best choice for spraying pesticide operation in the dispersed plots and hilly mountains. At present, extensive countries, ranging from Uruguay, Argentina, Brazil, to Australia are also using UAVs in agriculture to survey crop health, detect harvest readiness, and as a tool for surveying the damage from drought, flooding, weeds, and pests ${ }^{[5]}$. Japan has an estimated 10000 UAVs deployed for 
agricultural use, which cover $90 \%$ operations of the aerial crop dusting ${ }^{[6]}$; due to the small scale cultivation and hilly terrain, small helicopter aerial spray equipment is the leading spraying equipment in Japan ${ }^{[7]}$. While, from the current situation of China, fixed-wing aircraft has already become the main plant protection equipment in the agricultural reclamation areas of the Northeast and Xinjiang; application of multi-rotor UAV for plant protection field is also initially launched in the scattered farmland and hilly mountains of the Southern China ${ }^{[7,8]}$. Because of the poor working conditions in paddy network of Southern China, the traditional self-propelled and human-carrying land-spraying machine are inefficient and requires high intensity human labor ${ }^{[8]}$. The spraying operation of land-spraying machine leads to pesticide waste easily, it also threatens people's health and endangers the environment ${ }^{[9]}$. In addition, due to the inefficiency of land-spraying machine, timely and effective spraying application for crops nearly cannot be achieved in the pest and disease outbreaks season ${ }^{[10]}$. Therefore, the use of UAVs in plant protection has been developing rapidly in China, especially the aerial spraying application in Southern China ${ }^{[11]}$. Compared to fixed wing aircraft, the UAVs do not need specific takeoff and landing runway, it has low altitude flight performance $^{[12]}$. Additional, in comparison with traditional land-spraying machine, UAVs have the advantages of good mobility and high spraying efficiency $^{[13]}$.

As an emerging agriculture spraying equipment, there are still a lot of practical problems for UAVs spraying before popularization and application widely, such as low droplet coverage ratio, poor penetrability, ambiguous spraying operation parameters ${ }^{[14]}$. Recent studies ${ }^{[4,15,16]}$ indicate that the spraying parameters, such as nozzle configurations, release height and weather, not only impact the control effect against the pest and disease but also are closely related to the pesticide drift. In the analysis, control effect and drift are the different expression forms of movement for pesticide droplet in the coupled wind field. Drift of pesticide has uncertain impact on environment and human body, the efficiency and drift of aerial spray application are the major concern for the users, legislators and the public ${ }^{[1]}$. However, pesticide drift can't be absolutely avoided, extensive study has been conducted to predict and minimize drift ${ }^{[17]}$.

Generally speaking, spray drift is related to background atmospheric conditions, aircraft wing behavior and droplet size. Natural wind is the original aerodynamic factor which leads to aerial spray drift, while, drift occurs even when there is no wind, since wing rotating flow field plays a significant role in the behavior of the pesticide droplet after the release of pesticide ${ }^{[18]}$. Wing downwash flow field and wing vortices are the evolving consequence during the take-off, flight and landing process of UAVs. The counter rotating vortices produce up wash velocity areas near the wing tips where pesticide droplets are blown upwards beyond the wing tips, then it is prone to pesticide drift, whereas the wing downwash flow field happens under the wing tips, which can effectively enhance the deposition and reduce drift by moving pesticide droplets downwards crops. The deep study of tip interference and flow field distribution characteristics for multi rotor agricultural UAVs can provide the basis for the research on the movement law of droplets in the flow field, then the nozzle can be arranged at the better position in where the pesticide drift can be minimized.

In the western countries, after decades of field experimental and basic data accumulation, some models have been developed to predict the drift and deposition of aerial spray application, two of them are Forest Service Cramer-Barry Grim (FSCBG) and Agricultural Dispersal (AGDISP), which are still being constantly updated ${ }^{[19-22]}$. FSCBG was developed by Dumbauld et al. in $1980^{[23]}$, it is based on Gaussian model which is appropriate well for predicting long-range drift and simulating the effects of atmospheric stability, rather than the droplet distribution, deposition and drift. In contrast to FSCBG, the AGDISP $^{[24]}$ is the modified version based on Lagrangian approach that solves equations of wake vortex motion developed by $\operatorname{Reed}^{[25]}$. Wingtip interference characteristics of aerocraft are not considered in AGDISP $^{[26]}$, furthermore there is no model forecasting pesticide drift for small UAVs, and the AGDISP model also does not contain pesticide drift modular of the multi 
rotor UAVs. Variable nature of atmosphere conditions is so fleeting that the effects of them on the drift and dispersion in the aerial spraying application are difficult to assess with field experiment studies only. Therefore, it is quite necessary to study the intrinsic physical mechanism between flow field and the droplet transportation law under the influence of wing multi-rotor UAVs. The computational fluid dynamics (CFD) techniques happen to be used to achieve it, with witch the effects of variables on the drift and dispersion can be studied in isolation.

Nowadays, as the numerical analysis method, CFD has become a robust design tool in agriculture application $^{[27]}$. Spray drift potentials were simulated to improve sprayer performance ${ }^{[28-30]}$, and assist design of a pneumatic shielded spraying system was established for increasing spray deposition and reducing spray drift ${ }^{[31]}$. Considerable efforts of CFD simulation application have also been devoted to research the relative effects of droplet size, wind speed, turbulent intensity, initial droplet velocity, droplet discharge height, temperature and relative humidity on droplet displacement ${ }^{[32,33]}$ and collection efficiency ${ }^{[34]}$ in wind tunnel. CFD simulation has also been used to the aerial spraying field. The three dimensional near-field wake vortices of the Air Tractor AT-802 were simulated by Ryan et al. ${ }^{[35]}$, the results clearly showed that the significant entrainment of droplets exited in the aircraft's wingtip vortices, and droplets were moved outwards as well as lifted upwards by the wingtip vortices. The velocity field of Thrush 510 in the wake of fixed wing monoplane was studied in extreme ground effect with and without crosswind by Zhang et al. ${ }^{[1]}$ Zhang et al. ${ }^{[36]}$ simulated the pesticide drift condition of the N-3 unmanned helicopter spraying operation, and the results have some significance for the actual production.

Despite these preceding studies, the multi-rotor UAVs have been applied in aerial spraying field of China, while, synchronous research is lacking. The drift and deposition are affected by the crosswind, downwash air flow, and wing interference, which make up the content of the present investigation. As a preliminary step, the paper focuses on the velocity field of the UAV in hover, as well as its temporal evolution during spray application. The inclusion of sprayed droplets and their drift and deposition are scheduled for a subsequent paper.

\section{Working principle for six-rotor UAV}

The six-rotor UAV for simulation in this paper was provided by the Xi'an Wideworldz Aviation Science and Technology Co., Ltd, the SLK-5 plant protection UAV is shown in Figure 1.

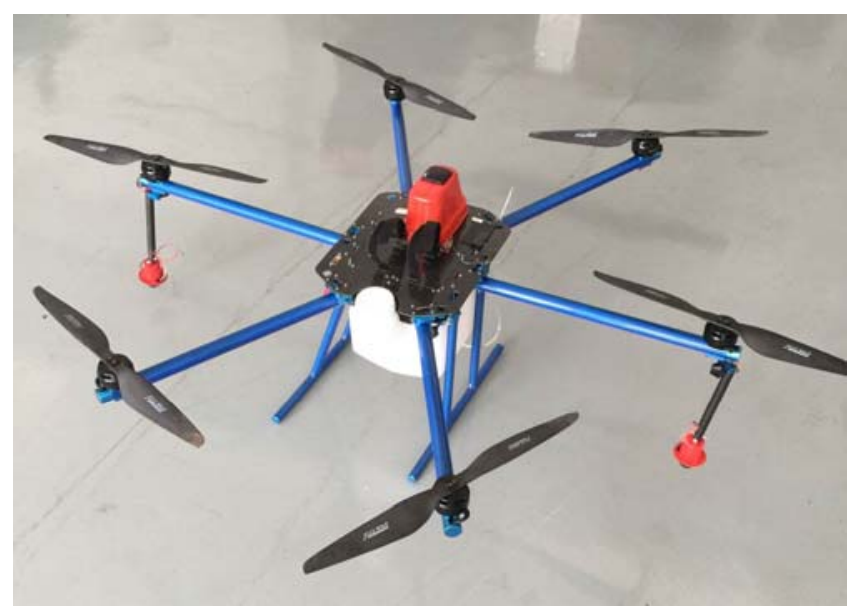

Figure 1 SLK-5 plant protection UAV

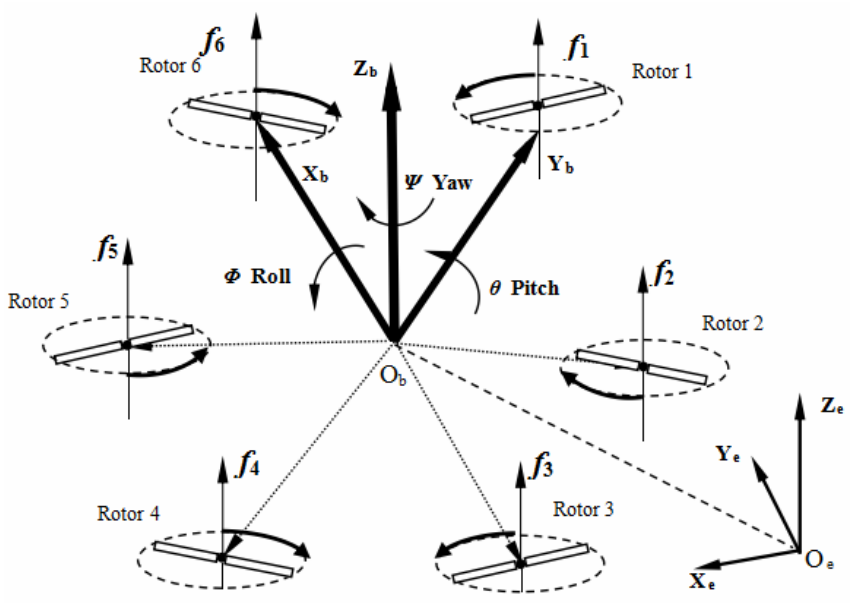

Figure 2 Six-axis UAV motion system

The speed of six-rotor UAV is relatively low, and the line speed, attitude and position can be measured based on ground point in the ground coordinate system $O_{e} X_{e} Y_{e} Z_{e}$ in Figure 2. The UAV body coordinate system $O_{b} X_{b} Y_{b} Z_{b}$ in Figure 2 is the base of rotorcrafts to be connected with each other and change with it. Six rotors are uniformly distributed along the circumferential direction, all rotor support arms are in same length and the included angle between any two arms is $60^{\circ}$, and rotation directions of the adjacent rotors are contrary, as shown in Figure 2. The $\Psi, \theta$ and $\Phi$ are the yaw angle 
rotating round the $Z_{b}$ axis, the pitch angle rotating round the $Y_{b}$ axis, the roll angle rotating round the $X_{b}$ axis separately ${ }^{[37,38]}$. Lifting force $\left(f_{i}, \quad i=1,2,3,4,5,6\right)$ generated by each motor is proportional to the square of the speed of the motor, that is $f_{i}=b \varpi^{2}$, so the flight attitude of the UAVs can be accomplished by adjusting the motor speed of the six motors.

Related to the aircraft attitude are up, down and hover. While increasing the speed of each rotor, the total lifting force is sufficient to overcome the gravity of the aircraft itself, and aircraft will rise. On the contrary, while reducing the rotational speed of each rotor, so that the total lifting force is less than gravity, the aircraft descended. Similarly, when the lift generated by the rotor and the body self gravity are equal, the UAVs will be in a hover state.

\section{Numerical simulation model}

\subsection{Numerical method}

Defined coordinate system in the inertial frame, the RANS equation of conservation integral form was established as the main control equation in which the effective parameters are the absolute physical quantities. Compared to the fixed (inertial) coordinate system, the RANS equation rotating (non-inertial) coordinate system attached a rotation due to the specific form of the source term to bring the equation can be expressed by Roberts and Murman ${ }^{[39]}$.

$$
\begin{gathered}
\frac{\partial}{\partial t} \iint_{\partial V o l} \boldsymbol{W} d V o l+\oiint[\boldsymbol{F}(\boldsymbol{W})-\boldsymbol{G}(\boldsymbol{W})] d \vec{S}=\iiint_{\partial V} \boldsymbol{Q} d V o l \\
\boldsymbol{W}=\left[\begin{array}{l}
\rho \\
\rho u \\
\rho v \\
\rho \omega \\
\rho E
\end{array}\right], \quad \boldsymbol{F}=\left[\begin{array}{l}
\rho\left(q_{n}-q_{b}\right) \\
\rho u\left(q_{n}-q_{b}\right)+p \hat{n}_{x} \\
\rho v\left(q_{n}-q_{b}\right)+p \widehat{n}_{y} \\
\rho \omega\left(q_{n}-q_{b}\right)+p \widehat{n}_{z} \\
\rho H\left(q_{n}-q_{b}\right)+p q_{b}
\end{array}\right],
\end{gathered}
$$

$$
\boldsymbol{G}=\left[\begin{array}{c}
0 \\
\tau_{x x} \hat{n}_{x}+\tau_{y x} \hat{n}_{y}+\tau_{z x} \hat{n}_{z} \\
\tau_{x y} \hat{n}_{x}+\tau_{y y} \hat{n}_{y}+\tau_{z y} \hat{n}_{z} \\
\tau_{x z} \hat{n}_{x}+\tau_{y z} \hat{n}_{y}+\tau_{z z} \hat{n}_{z} \\
\Phi_{x} \hat{n}_{x}+\Phi_{y} \hat{n}_{y}+\Phi_{z} \hat{n}_{z}
\end{array}\right], \quad \boldsymbol{Q}=\left[\begin{array}{c}
0 \\
\rho \omega \Omega \\
0 \\
-\rho \mu \Omega \\
0
\end{array}\right]
$$

where, $\boldsymbol{W}$ is the conserved variable vector; $\boldsymbol{F}(\boldsymbol{W})$ and
$\boldsymbol{G}(\boldsymbol{W})$ are non viscous flux and viscous flux respectively, $\boldsymbol{Q}$ is the source term caused by rotor rotation; $\rho$ and $p$ and are the density and pressure of the fluid; $u, v, \omega$ are fluid velocity components; $E$ is the internal energy of a fluid unit; $\boldsymbol{n}=\left[\hat{n}_{x}, \hat{n}_{y}, \hat{n}_{z}\right]$ denotes the normal vector of the control surface; Vol denotes the unit volume, and $\Omega$ denotes rotating speed of rotor; $q_{n}=u \hat{n}_{x}+v \hat{n}_{y}+\omega \hat{n}_{z}$ and $q_{b}=u_{b} \hat{n}_{x}+v_{b} \hat{n}_{y}+\omega_{b} \hat{n}_{z} \quad$ denote fluid velocity and speed grid along the normal component of mesh surface respectively; $\tau_{x x / y y / z z}, \tau_{x y / x z / y z}$ and $\Phi_{x / y / y}$ are the related viscous quantities; $\mu, k, T$ denote viscosity coefficient, thermal conductivity and absolute temperature respectively.

Viscous force is not negligible in the numerical simulation of rotor rotating wind field, otherwise it will produce a significant deviation. At present, the RANS equation has been widely used in numerical simulation of rotor flow field ${ }^{[40]}$. In this paper, we choose the RNG $k$ - $\varepsilon$ turbulence model which is suitable for both high Reynolds number and low Reynolds number ${ }^{[41]}$.

In this paper, the finite volume method was used to discrete the governing equations of the flow field. In order to improve the clarity of the flow field, the convection field was calculated by using the coupling algorithm under the double precision mode. The solving process of coupling algorithm is shown in Figure 3.

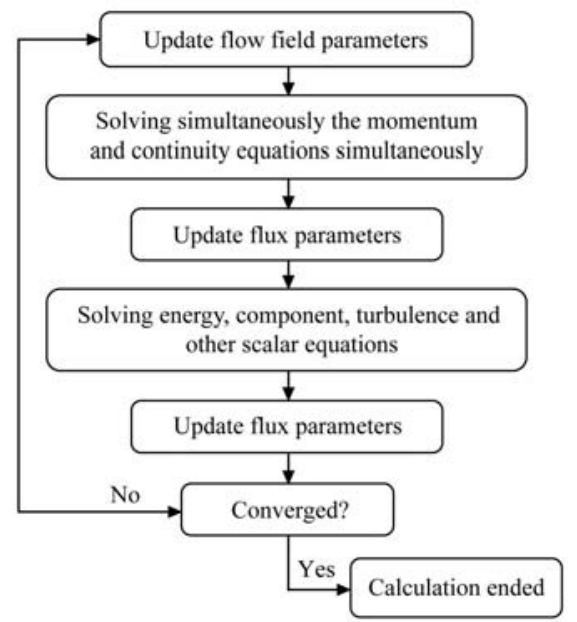

Figure 3 Calculation flowchart of coupling algorithm

\subsection{Model description}

The computational domain was defined as a cylinder, its radius is $2 \mathrm{~m}$, and the column height is $4.1 \mathrm{~m}$. Additionally, the center coordinates of the cross-section 
of the wing, upper and lower surfaces of the computational domain are $(0,0,0.426),(0,0,-0.124)$, and $(0,0,3.976)$ respectively, as shown in Figure 4 . It should be noted that the radial distance between the rotating center of the wing and center axis of the computational domain was $0.55 \mathrm{~m}$.

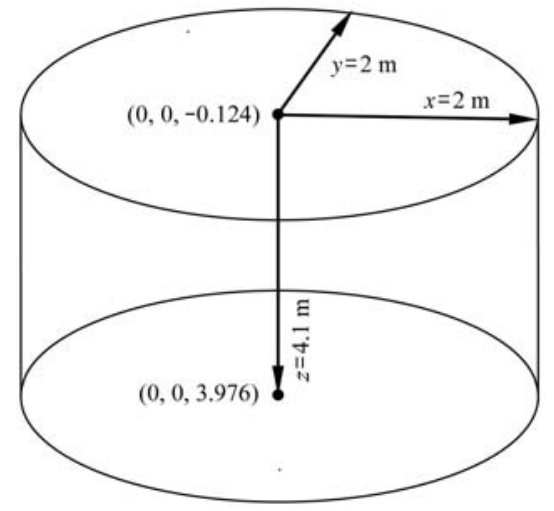

Figure 4 Calculation area diagram schematic diagram

In the SLK-5 plant protection UAV, rotor is the most important part. Therefore, three dimensional Optimscan5-2015011K05 provided by Manfrotto was used to scan the rotor, then the $3 \mathrm{D}$ modeling of the rotor was obtained by using the Geomagic software to deal with the point cloud, as shown in Figure 5.

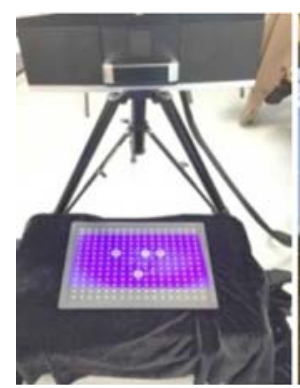

a. Calibration plate

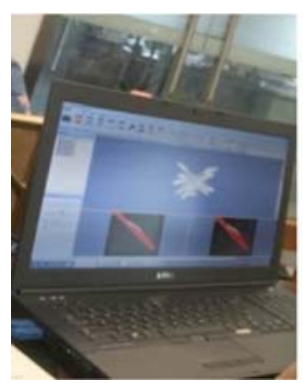

d. Locating surface

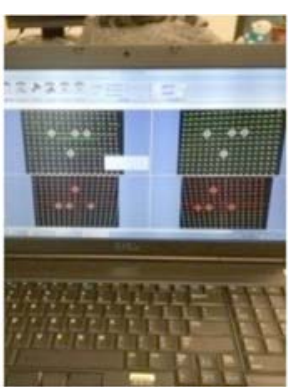

b. Calibration Process

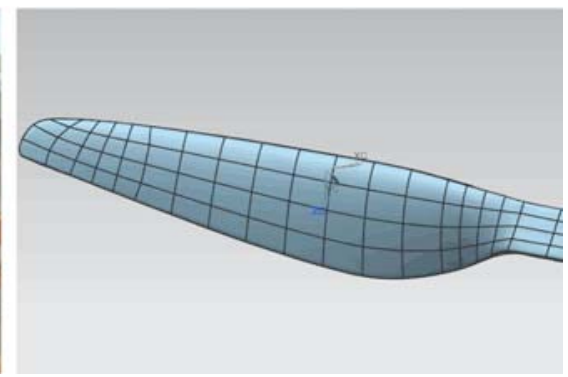

e. Point cloud synthesis

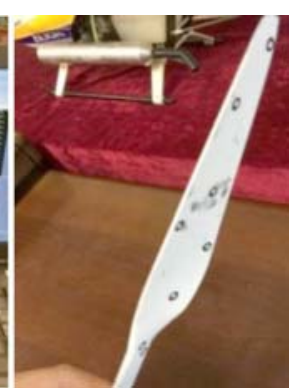

c. Wing mark
Figure 5 Wing scanning and point cloud processing

Several components of UAV were not considered in the three-dimensional numerical model, including the landing gear and cantilever. Due to lower height of the landing gear and long distance between the wings and fuselage, this simplification has little effect on the development and evolution of the downwash airflow. A grid sensitivity study was carried out. Three sets of grid (coarse, medium, and fine) were generated, with the grid number spacing ranging from 3.2 million, 5.6 million and 720 million. Grid sensitivity study results showed that 3.2 million was enough to capture the flow field evolution process. To ensure higher accuracy, the medium grid was chosen to perform the simulations which were used to simulate field conditions. Hexahedral structured grid scheme was adopted to mesh all the computational domain, and the total number of grid reached 5.6 million finally. In the numerical simulation model, no slip adiabatic wall boundary was adopted for all the solid wall. In addition, in the hovering state, the speed of the wing is $288 \mathrm{rad} / \mathrm{s}$.

\section{Downwash airflow speed test}

The SLK-5 plant protection machine was hovering at the vertical height of $3.55 \mathrm{~m}$ above the ground. The Kestrel 4500 Pocket Weather Tracker, fixing on the top of the movable platform, was used to measure the wind vertical velocity of the marked points, as shown in the Figures 6 and 7. The center coordinates of the two tested surfaces are $(0,0,1.426)$ and $(0,0,2.426)$. The marking points on the tested surfaces are Point 1/2-1, Point 1/2-2, Point 1/2-3, Point 1/2-4, Point 1/2-5, Point 1/2-6, under the 6 rotors of the SLK-5 plant protection machine at the vertical height of $1 \mathrm{~m}$ and $2 \mathrm{~m}$ from the wing, as shown in Figure 8 . The meteorological conditions were also measured and recorded, such as temperature, barometric pressure, and horizontal wind speed, which were $35^{\circ} \mathrm{C}, 94430 \mathrm{~Pa}, 0.2 \mathrm{~m} / \mathrm{s}$, respectively. These data would be used as boundary conditions for the numerical computation.

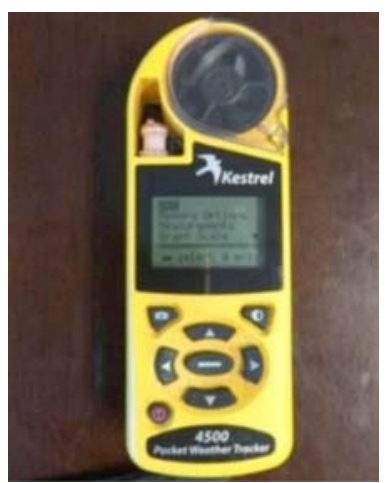

Figure 6 Pocket weather tracker 

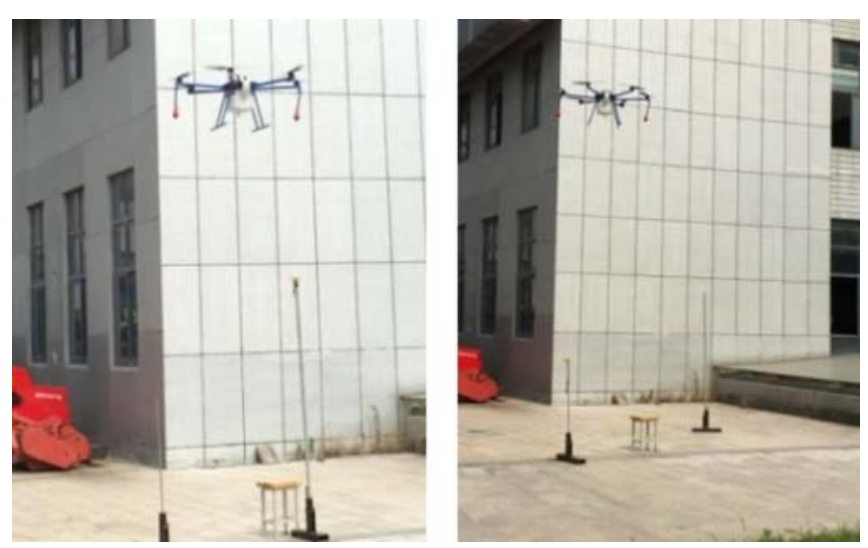

Figure 7 Wind velocity test experiment photos

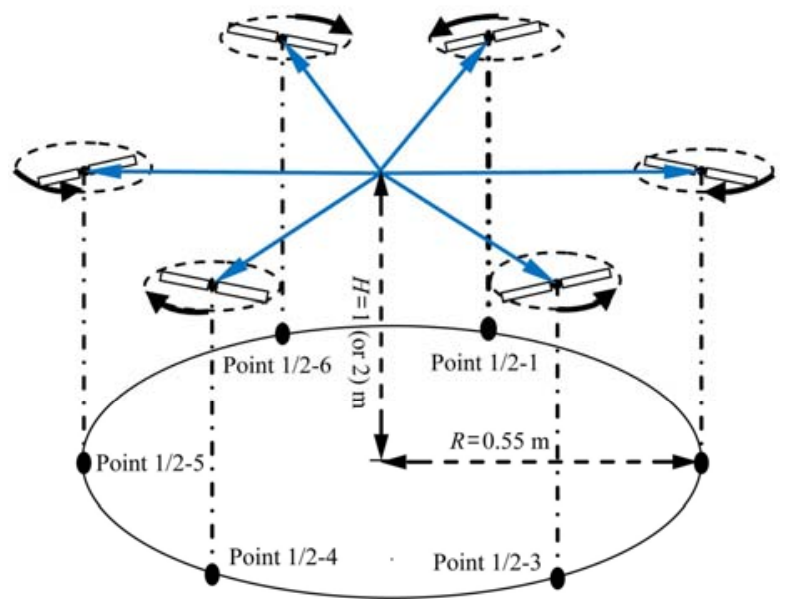

Figure 8 Distribution of wind velocity measurement points

Table 1 shows the experimental measurements and simulation values of the $z$-direction velocity for the marked points, the relative error between the two values is also given, and the overall situation on the numerical calculation will be described later.

Table 1 Experimental measurements and simulation values of the $z$-direction velocity for the marked points

\begin{tabular}{|c|c|c|c|c|c|c|c|}
\hline \multicolumn{2}{|c|}{$\begin{array}{l}\text { Wind } z \text {-direction velocity } \\
\text { magnitude, } \mathrm{m} \cdot \mathrm{s}^{-1}\end{array}$} & Rotor 1 & Rotor 2 & Rotor 3 & Rotor 4 & Rotor 5 & Rotor 6 \\
\hline \multirow{3}{*}{$\begin{array}{l}\text { Vertical } \\
\text { height } \\
1 \mathrm{~m} \text { from } \\
\text { the wing }\end{array}$} & $\begin{array}{l}\text { Experimental } \\
\text { value }\end{array}$ & 8.1 & 7.8 & 8.2 & 8.1 & 7.9 & 7.9 \\
\hline & $\begin{array}{l}\text { Calculated } \\
\text { value }\end{array}$ & 8.85 & 8.80 & 8.83 & 8.85 & 8.87 & 8.83 \\
\hline & $\begin{array}{l}\text { Absolute } \\
\text { relative error }\end{array}$ & $8.5 \%$ & $11.3 \%$ & $7.1 \%$ & $8.4 \%$ & $10.9 \%$ & $10.5 \%$ \\
\hline \multirow{3}{*}{$\begin{array}{l}\text { Vertical } \\
\text { height } \\
2 \mathrm{~m} \text { from } \\
\text { the wing }\end{array}$} & $\begin{array}{c}\text { Experimental } \\
\text { value }\end{array}$ & 4.5 & 4.6 & 4.4 & 4.5 & 4.5 & 4.6 \\
\hline & $\begin{array}{l}\text { Calculated } \\
\text { value }\end{array}$ & 4.92 & 5.06 & 4.98 & 4.83 & 4.90 & 5.03 \\
\hline & $\begin{array}{l}\text { Absolute } \\
\text { relative error }\end{array}$ & $8.5 \%$ & $9.1 \%$ & $11.6 \%$ & $6.8 \%$ & $8.2 \%$ & $8.5 \%$ \\
\hline
\end{tabular}

From the Table 1, we can see that the relative error of the numerical value is less than $12 \%$ compared to the experimental value. Taking the weak atmospheric turbulence interference into account, the accuracy of numerical simulation is acceptable. Through the experiment, only the wind speed of some points can be measured, while the experiment appears powerless to the description of the flow pattern and flow characteristics of the down wash flow. So, we can see that numerical simulation appears very important.

\section{Numerical simulation of downwash air flow in hover}

The velocity distribution of the $x$-, $y$-, and $z$-directions on the $y o z$ plane are presented in Figure 9 after the full development of the downwash airflow at $2.3722 \mathrm{~s}$. As it can be seen from the Figure 9, in the development process, a clear discontinuity phenomenon happened for the downwash air flow at $1.6272 \mathrm{~s}$. It also shows that the max velocity value of the $z$-direction is close to $9.6 \mathrm{~m} / \mathrm{s}$ at $2.3722 \mathrm{~s}$, clearly the largest proportion before the downwash airflow develops to the ground. With the development of downwash airflow, the velocity of $x$-direction and $y$-direction gradually declined to 0 . While stagnation occurred to the velocity of airflow in the $z$-direction, and airflow direction changed to the $y$-direction, then the velocity magnitude of $y$-direction increased suddenly.

Due to the existence of turbulence and the disturbance of rotor rotation, the downwash airflow air flow was not stable, and breaking phenomenon occurred in the middle of downwash airflow. As shown in Figures 9c and 9f, the velocity distribution of $z$-direction indicated that the velocity at airflow broken part was negative direction. We could infer the downwash airflow instability led to the emergence of the vortex structure.

The research on the distribution law of the downwash airflow, the development process of the flow field, and the flow attenuation mechanism could be of great significance to deepen the understanding of the pesticide drift and improve the effect of inhibiting drift. Six rotors are uniformly distributed along the circumferential direction, and rotation directions of the adjacent rotors are contrary, as shown in Figure 10, the flow field has a certain period symmetry. So, Figure 11 shows the total velocity distribution disciplinarian of the two typical cross sections yoz and $x o z$ planes. In the main section of the yoz plane, the down wash flow presents good 
symmetry, after the transport of $3.55 \mathrm{~m}$, the velocity of the down wash flow is reduced from $10 \mathrm{~m} / \mathrm{s}$ to less than $5 \mathrm{~m} / \mathrm{s}$. Rotor high-speed rotation makes the flow velocity of inner circle is also increases, resulting in the pressure reduction in the inner circle. So, in conjunction with Figure 12, we can see that it appears a significant "shrinkage characteristic" in the downwash flow under the extrusion of the atmospheric pressure.

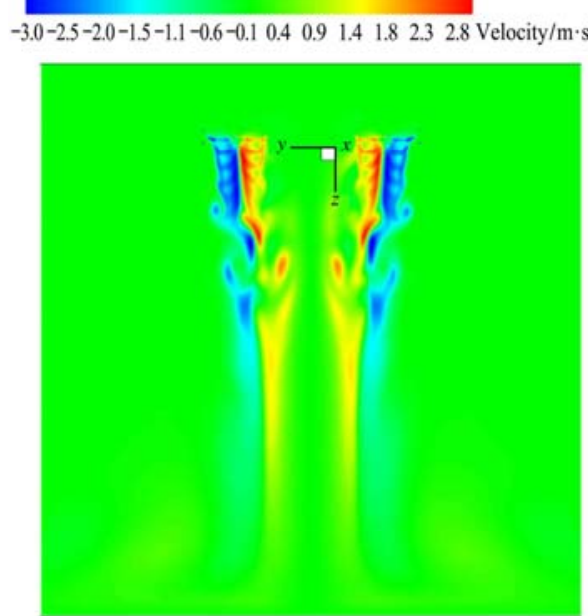

a. $x$-direction

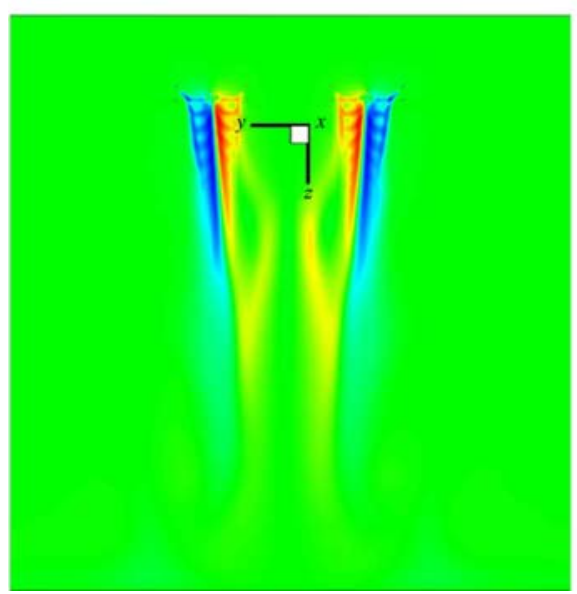

d. $x$-direction

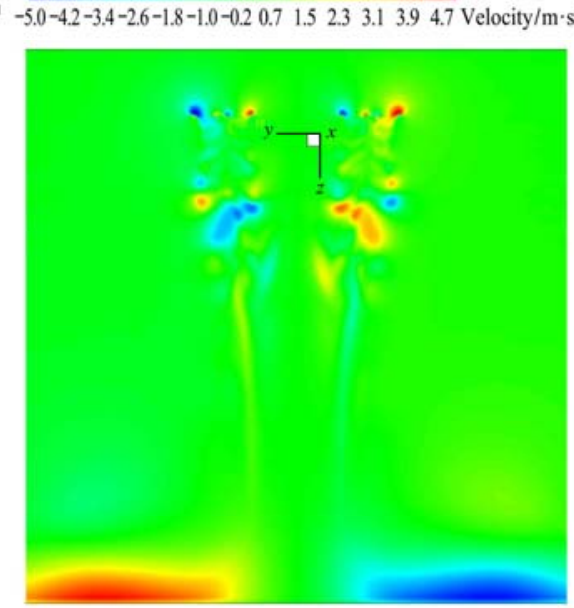

b. $y$-direction

$t=1.6272 \mathrm{~s}$

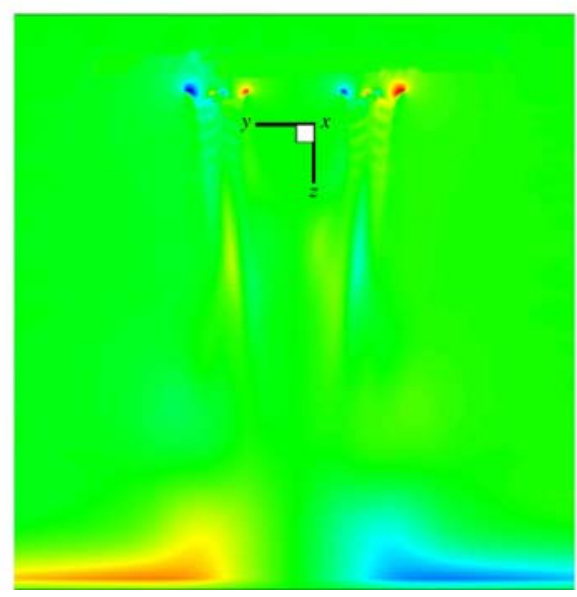

e. $y$-direction $t=2.3722 \mathrm{~s}$
$-2.0-1.0-0.10 .91 .92 .83 .84 .85 .86 .77 .78 .79 .6$ Velocity $/ m \cdot s^{-1}$

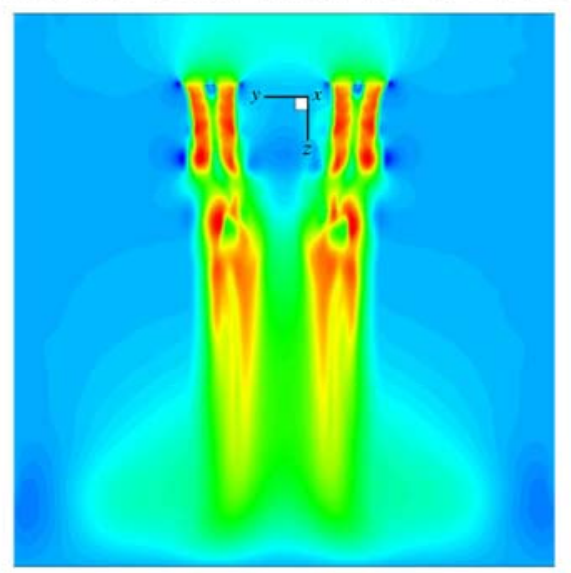

c. $z$-direction

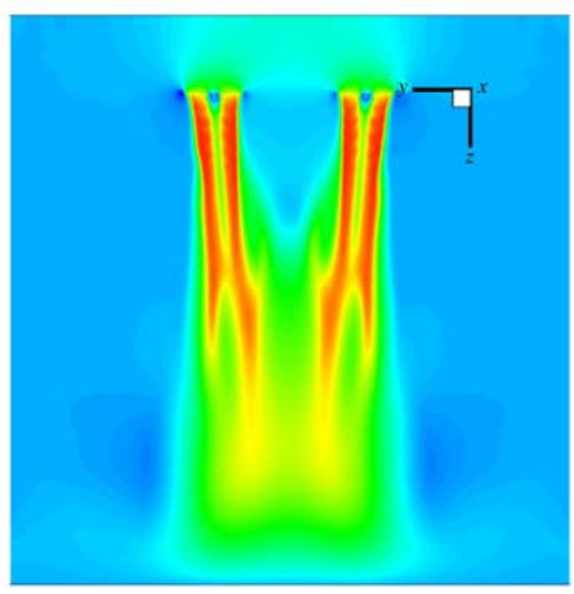

f. $z$-direction

Figure 9 Velocity distribution in three directions of $Y O Z$ plane

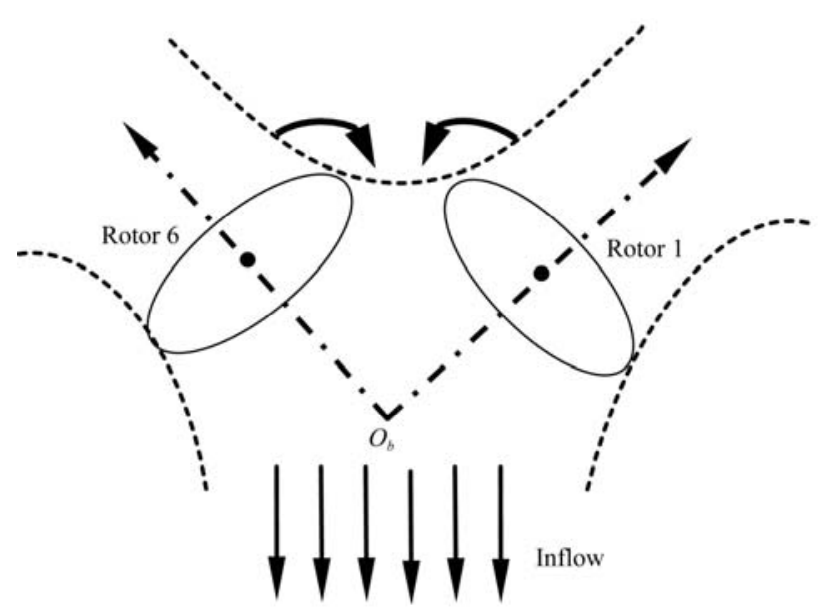

a. Inflow

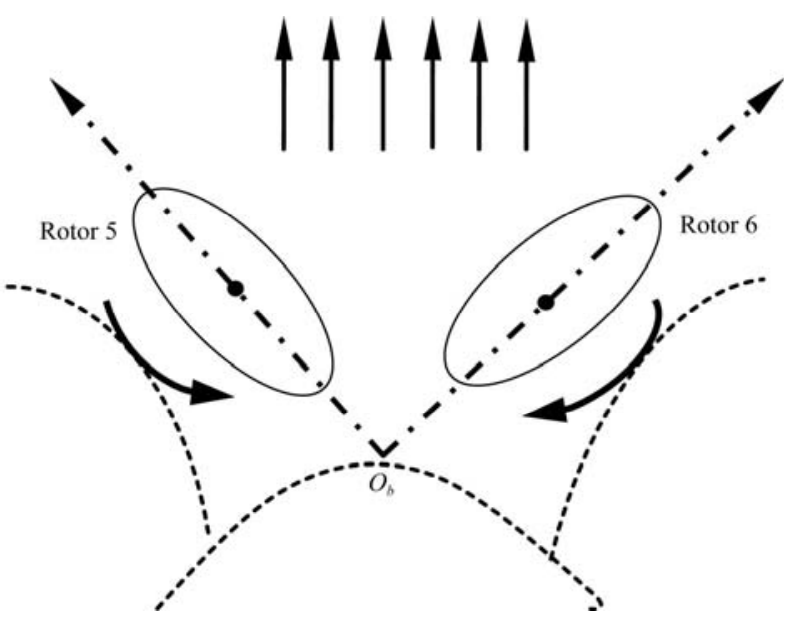

b. Outflow

Figure 10 Two motion states of adjacent wings 


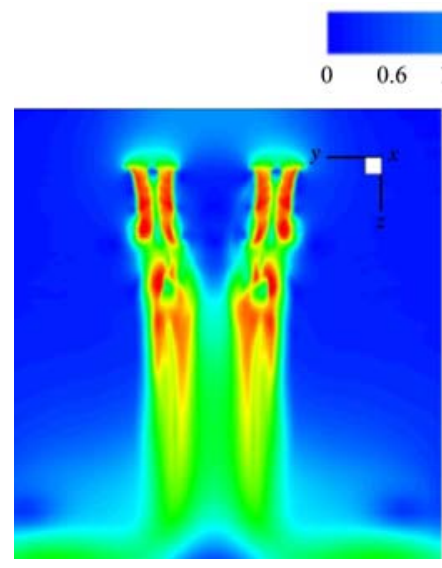

a. $t=1.6272 \mathrm{~s}$, yox plane

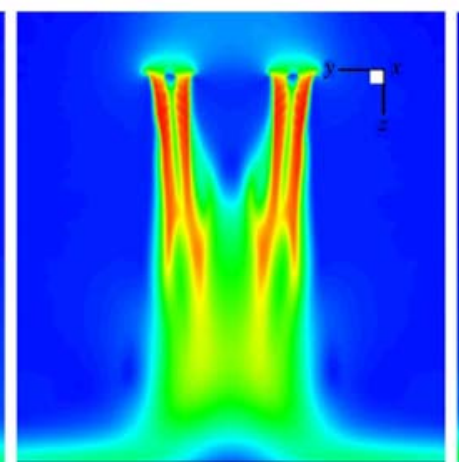

b. $t=2.3722 \mathrm{~s}, y o z$ plane

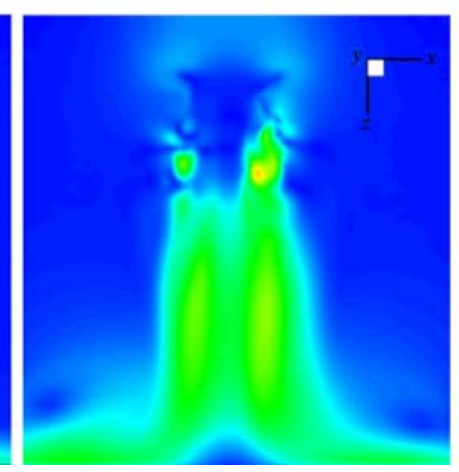

c. $t=1.6272 \mathrm{~s}, x o z$ plane

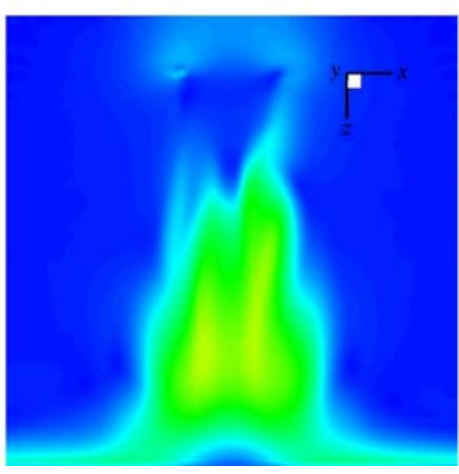

d. $t=2.3722 \mathrm{~s}, y o z$ plane

Figure 11 Total velocity magnitude distribution of two planes

$P / \mathrm{Pa}$

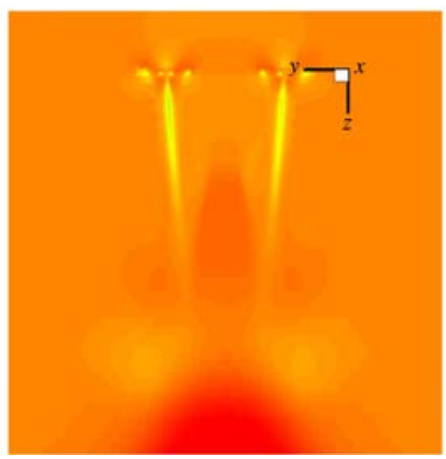

b. $t=2.3722 \mathrm{~s}, y o z$ plane

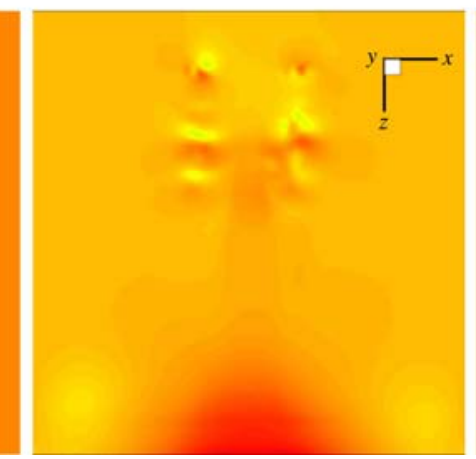

c. $t=1.6272 \mathrm{~s}, x o z$ plane

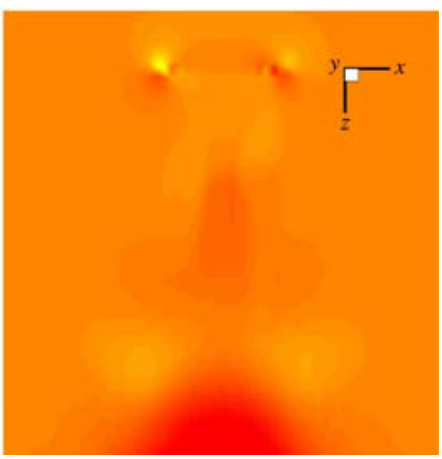

d. $t=2.3722 \mathrm{~s}, x o z$ plane

Figure 12 Pressure distribution of two planes

In Figure 11, there is a "significant asymmetry" in the velocity distribution of the $x o z$ plane. Figure 10 shows the rotation law of adjacent wing, the rotation directions of rotor 1 and rotor 6 are opposite, while both the two rotors induce flow into the inner circle. On the contrary, the rotor 5 and rotor 6 induce flow to the outer circle. So we can see from the $x o z$ plane in the Figure 9, the velocity of the $x$ negative direction is significantly less than the velocity of $x$ positive direction under the vertical regions not far from the rotor. Along with the increase of distance, the influence of the wing inducement is weakened, the velocity of the $x o z$ plane tends to be uniform. Figure 12 shows the pressure distribution of the downwash airflow. As shown in Figure 12, under the disturbance of the wing and the pressure of the external atmosphere, some vortexes appeared in the downwash airflow. Air pressure is significantly reduced in the vortex region. "Stagnation phenomenon" happens after the downwash airflow develops to the ground, then air pressure increased obviously.

Figure 13 shows the distribution law of the total flow velocity in the $z$-direction of each typical height section. It can be seen from the Figure 13, high speed regions and low speed areas are quite distinct from each other, especially in the areas near the wing. The high speed areas focused on the region under the wing, which are symmetrical distribution of 6 circular areas. With the vertical distance from the rotor farther and farther, the high speed areas gradually reduced, the maximum velocity also showed a trend of decline. From the Figure 13f, we can see that in the plane of $z=3.426$, the wind speed difference in the core area of the down wash air is significantly decreased.

Combined Figure 14a and Figure 11, under the suction of the next wash, the external pressure is greater than the air pressure in the lower air flow area, peripheral airflow also flows to the central area of the downwash air flow. By comparing Figure $14 \mathrm{~b}$ and Figure 13, a conclusion can be drawn that if the two adjacent wings induce airflow to the rotor inner circle, then the maximum air velocity value between the two wings is obviously larger. 


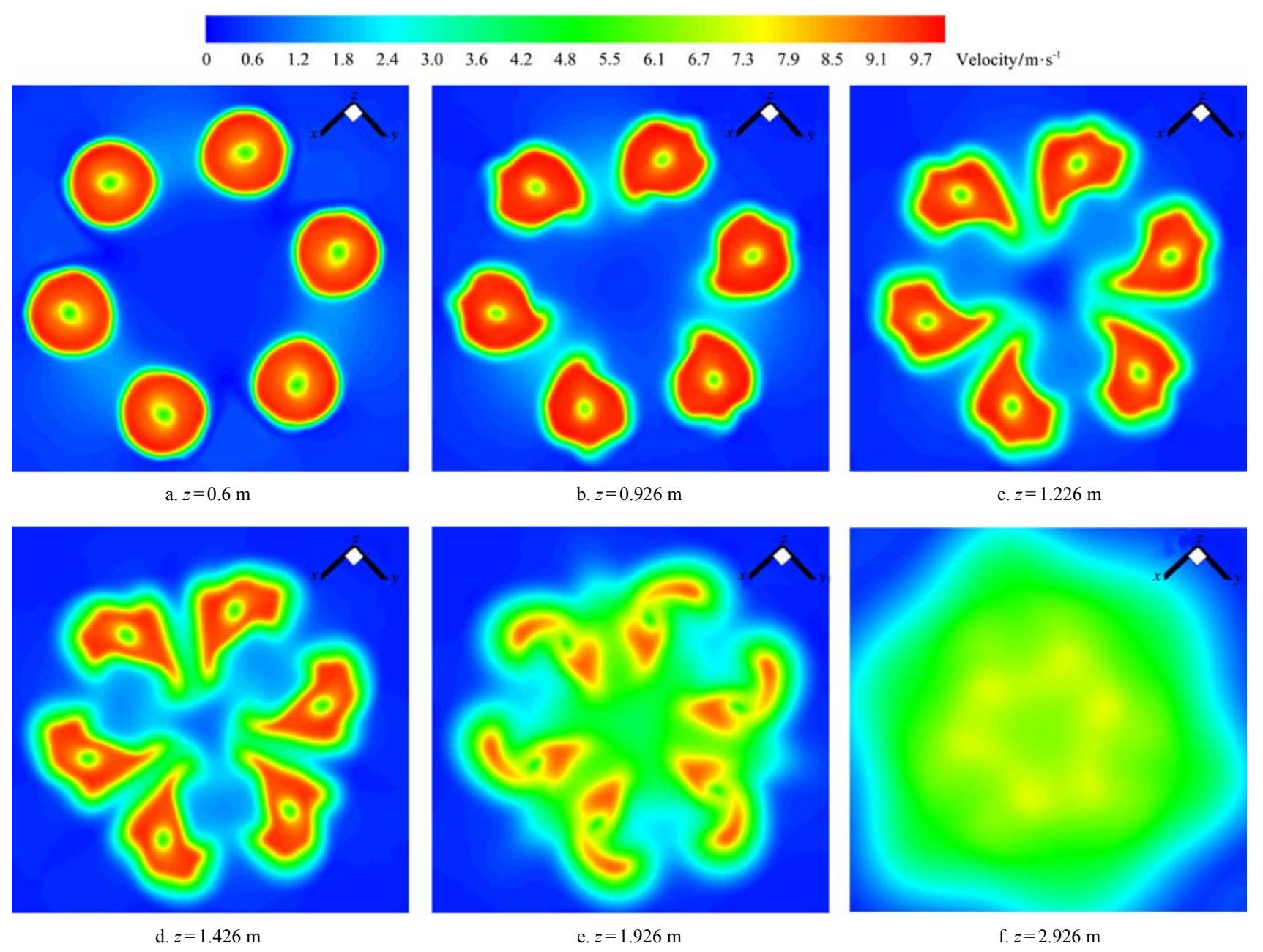

Figure 13 Velocity magnitude distribution of xoy plane at different heights for $z$-direction $(t=2.3722 \mathrm{~s})$

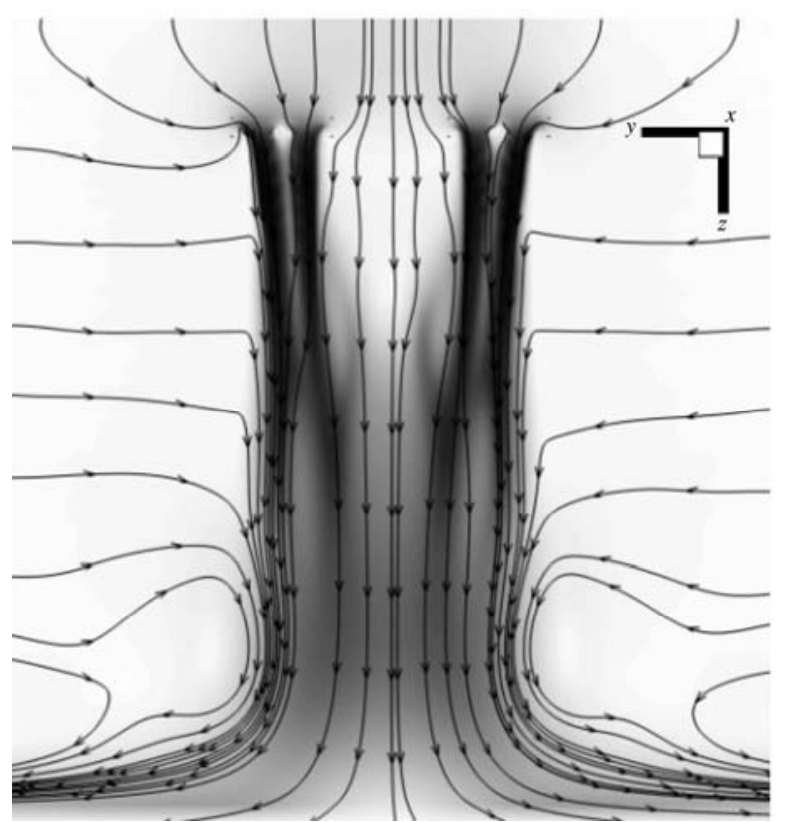

a. $y o z$ plane $t=2.3722 \mathrm{~s}$

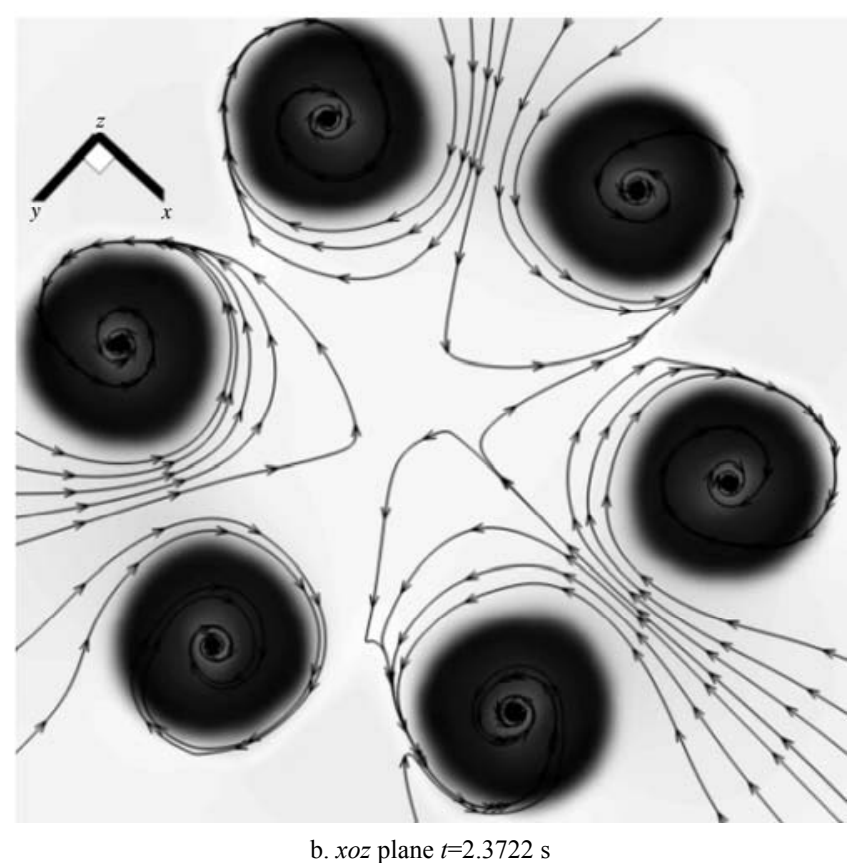

b. $x o z$ plane $t=2.3722 \mathrm{~s}$

Figure 14 Streamline diagram of $y o z$ and $x o z$ planes $(z=0.6 \mathrm{~m})$

In the previous description, the flow pattern and velocity distribution of the down wash flow are analyzed qualitatively. In order to make a more intuitive research on the distribution of the downwash air and characteristic of wing interference, Figures 15 shows the velocity distribution along the $y$-direction of different heights on the two typical cross sections, the $y o z$ plane and $x o z$ plane. There are two kinds of flow states between adjacent 
wings, the inflow and outflow, as shown in Figure 12, and this is the root cause of the interference between the wings. As can be seen from Figure 15a, the air velocity has two peaks along the y direction, while the two peaks are completely different at different heights. With the distance from the wings getting farther and farther, the difference of the two peaks at the same height is growing, and then gradually tend to unify, while the maximum peaks of different heights are gradually increased and then gradually shrink. The cause of this phenomenon can be analyzed from the following process. We know that Figure 15a and $x o z$ plane in Figure 12 are corresponding to each other. By comparing the two Figures, it can be seen that wing rotating induction is the dominant factor for the velocity distribution, while wing interference phenomenon is not obvious; with the distance from the wings getting farther, the interference

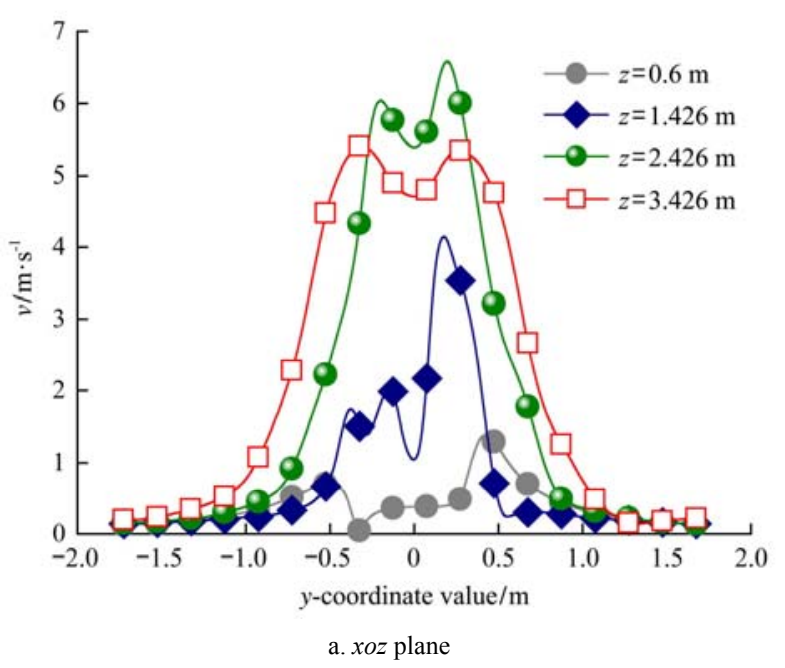

effect caused by the inconsistency between the wings is increasing; with the further increase of the distance, the influence of the wing on the downwash flow is reduced, and the two peaks of the two velocity on the specific plane are also uniform.

Combined with Figure 10b and Figure 15b, it can be seen that the air velocity has nearly two symmetrical peaks along the $y$-direction on the $y o z$ plane. With the increase of the distance from the wing, the highest peak of air velocity decreases gradually. Another point of concern is that the distance between the two peaks is getting closer and closer. The above phenomenon can be explained from the following two aspects: with the distance from the wings getting farther and farther, the downwash air flow gradually weakened; and the downwash airflow shrinks inward under the extrusion of outer circle atmospheric pressure at the same time.

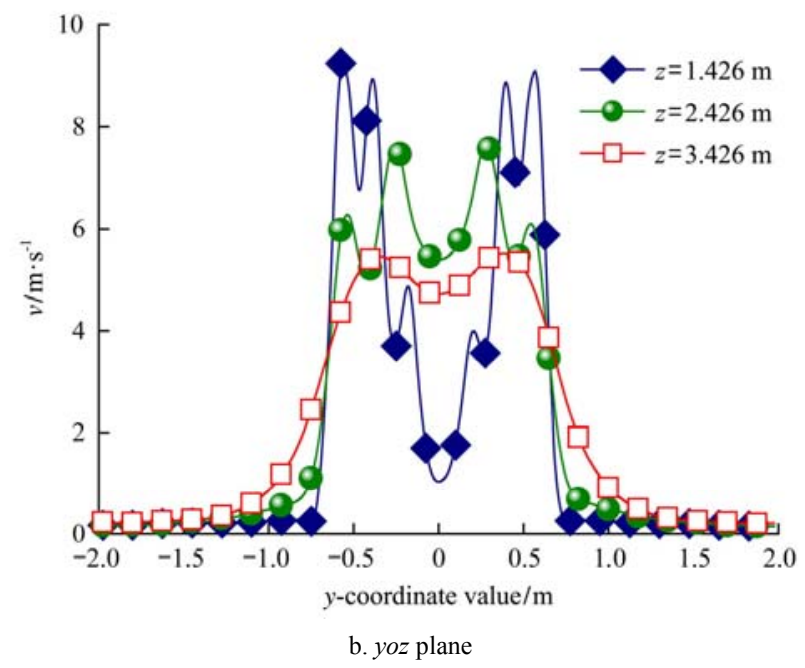

Figure 15 Velocity magnitude at different heights

Figures $16 \mathrm{a}$ and $16 \mathrm{~b}$ show the velocity components of $x$-, $y$-, and $z$-directions along the $y$-direction at the height of $z=1.426 \mathrm{~m}$ and $z=3.426 \mathrm{~m}$ on the $x o z$ plane. As can be seen from Figure 16a, in the middle of the downwash air flow, the effects of wing interferences on the velocity of the three directions are very severe. Combined with Figure 16a and Figure 14, it can be seen that superposition of the three directional wing effects leads to a huge difference between the two peaks. Figure 16b shows that the influence of wing interference on the velocity distribution is gradually weakened when the distance from the wing reaches a certain level.
Figures $16 \mathrm{c}$ and $16 \mathrm{~d}$ show the velocity components of $x$-, $y$-, and $z$-directions along the $y$-direction at the height of $z=1.426 \mathrm{~m}$ and $z=3.426 \mathrm{~m}$ on the $y o z$ plane. From the two figures, the velocity components of $x-, y$-, and $z$-directions are substantially symmetrical. With the distance from the wings getting farther and farther, velocity of the three directions all appear different degrees of attenuation, and the velocity peak of $z$-direction is the most obvious. However, with the diffusion of the down wash flow, velocity in local area of $z$-direction increases, and the range of effective action for downwash air flow is further increased. 


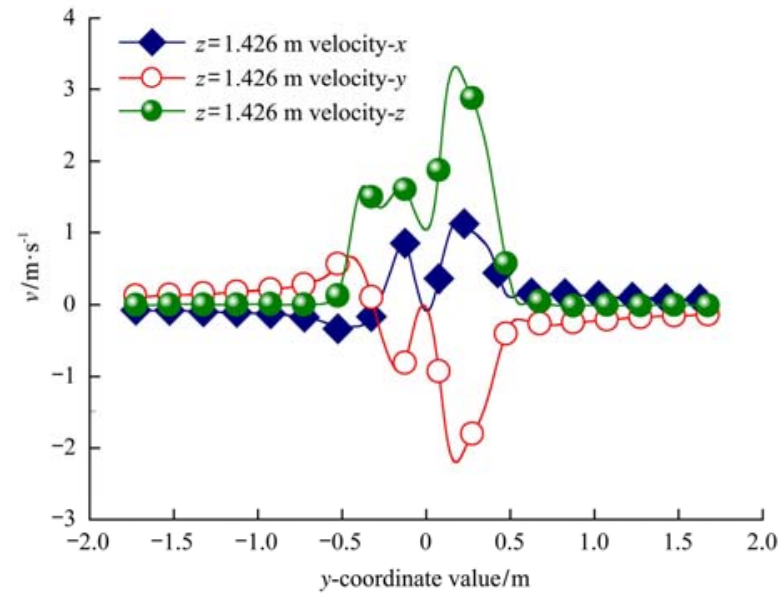

a. $x o z$ plane at the height of $z=1.426 \mathrm{~m}$

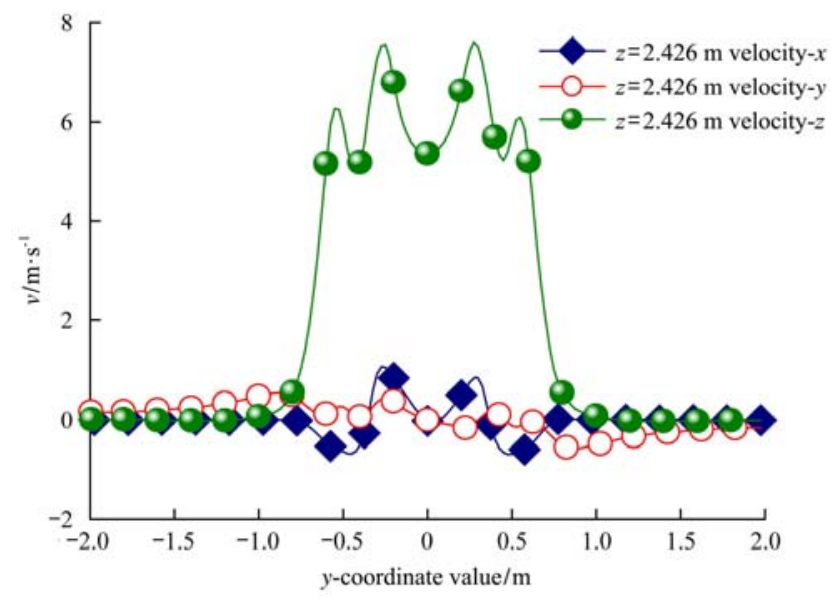

c. yoz plane at the height of $z=1.426 \mathrm{~m}$

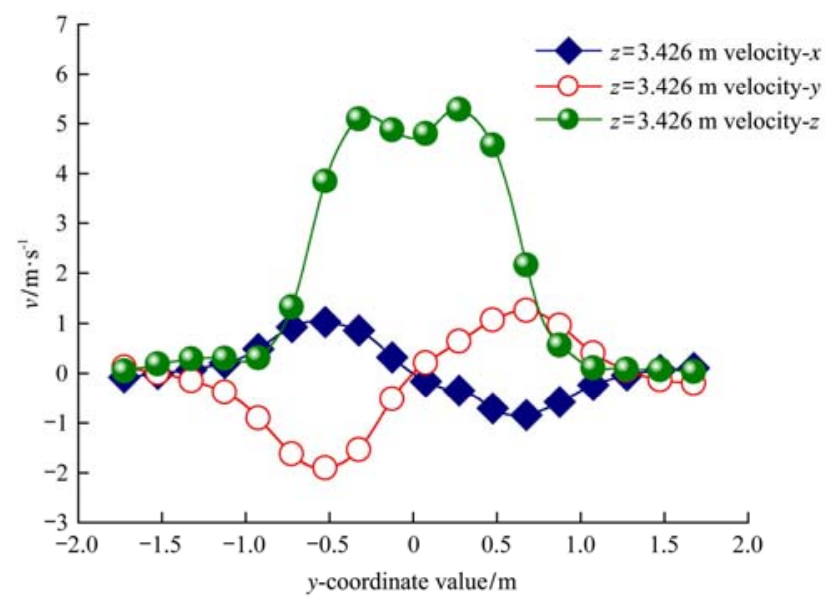

b. $x o z$ plane at the height of $z=3.426 \mathrm{~m}$

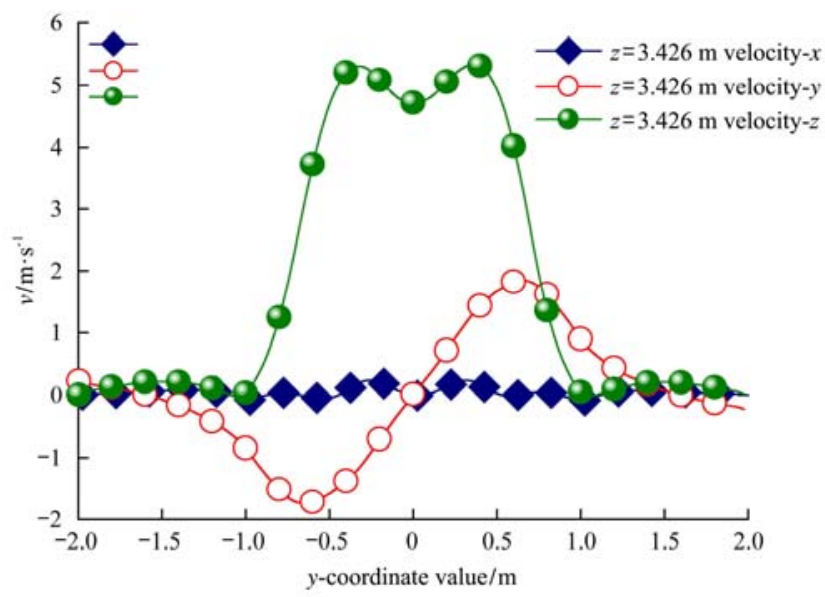

d. yoz plane at the height of $z=3.426 \mathrm{~m}$

Figure 16 Velocity distribution in three directions of different planes at the distinct height

\section{Conclusions}

For the application of UAVs in plant protection field, obtaining the distribution law of the unique downwash air flow is the key step to control and improve spraying quality. The downwash airflow of the SLK-5 plant protection UAV in hover was studied based on the numerical simulation and wind velocity test. The following conclusions were obtained.

(1) Based on compressible Reynolds-averaged Navier-Stokes (RANS) equations with the RNG $\boldsymbol{k}-\boldsymbol{\varepsilon}$ turbulence model, the efficient three-dimensional computational fluid dynamics (CFD) method was established. Downwash airflow of the SLK-5 plant protection UAVs in hover was simulated.

(2) Combined with wind velocity test experiment, reliability and validity of numerical calculations has been verified. The comparison results show that the relative error is less than $12 \%$ between the experimental and simulated values of the $z$-direction velocity at the marked points.

(3) The velocity distribution law obtained by the numerical simulation shows that the $z$-direction velocity is dominant in the main area of the down wash air flow. After the down wash flow developed to the ground, stagnation occurred to the airflow of $z$-direction velocity component decays significantly, and $y$-direction velocity component increases obviously in the yoz section.

(4) The maximum velocity value of the downwash flow is close to $10 \mathrm{~m} / \mathrm{s}$. The influence of wing interference on the $x, y, z$ velocity components distribution is so significant in the longitudinal section between two wings that the two velocity peaks in the section are completely different, especially in the middle area of the downwash flow. While the velocity components of $x$-, $y$-, and $z$-directions are substantially symmetrical at different height on the yoz plane. With the distance from the wings getting farther and farther, 
downwash air flow gradually weakens, and the velocity distribution tends to be uniform on the xoy radial cross section.

(5) In order to avoid the effects of wing interference on pesticide application, it is suggested that the working height from the crop top of the SLK-5 plant protection UAVs should be about $0.6 \mathrm{~m}$.

\section{Acknowledgements}

The authors acknowledge the financial support provided by the National Key Research and Development Plan of China (No. 2016YFD0200702); Study on Key Techniques of Aviation Plant Protection for Rice Diseases and Insect Pests of China (No. S201729); Open exchange project of China-US pesticide technology Joint Research Center (No. Y2017PT32); Aviation intelligent pesticide operation system based on Beidou automatic navigation (No. S201609).

\section{[References]}

[1] Zhang B, Tang Q, Chen L P, Xu M. Numerical simulation of wake vortices of crop spraying aircraft close to the ground. Biosystems Engineering, 2016; 145: 52-64.

[2] John H. Unmanned Flight: The Drones Come Home. National Geographic, March 2013.

[3] Yang Y S, Zhang J P, Nie S L. Energy loss of nozzles in water jet system. J. Mech. Eng., 2013; 49(2): 139-145. (in Chinese).

[4] Matthew G. Rise of the Ag Drones. Precision Agr. 2013; March 6.

[5] Green M. Unmanned drones may have their biggest impact on agriculture. Daily Beast, 2013

[6] Jenkins D, Bijan V. Association for Unmanned Vehicle Systems International. The economic impact of unmanned aircraft systems integration in the US. Robotics Tomorrow, 2013.

[7] Xue X Y, Liang J, Fu X M. Prospects of China's aviation plant protection technology. Chinese Agricultural Mechanization, 2008; 5: 72-74. (in Chinese)

[8] Xue X Y, Tu K, Qin W C, Lan Y B, Zhang H H. Drift and deposition of ultra-low altitude and low volume application in paddy field. Int. J. Agric. Biol. Eng., 2014; 7(4): 23-28.

[9] Zhang W J, Jiang F B, Ou J F. Global pesticide consumption and pollution: with China as a focus. In: Proceedings of the International Academy of Ecology and Environmental Sciences, 2011; 1, pp. 125-144.
[10] Sogawa K. The rice brown plant hopper: feeding physiology and host plant interactions. Annu. Rev. Entomol., 1982; 27(1): 49-73.

[11] Xue X Y, Liang J, Fu X M. Prospect of aviation plant protection in China. Chin. Agric., 2008; 5: 72-74. (in Chinese)

[12] Zhang D Y, Lan Y, Chen L P, Wang X, Liang D. Current status and future trends of agricultural aerial spraying technology in China. Transactions of the CSAM, 2014; 45(10): 53-59. (in Chinese)

[13] Bae Y, Koo Y M. Flight attitudes and spray patterns of a roll-balanced agricultural unmanned helicopter. Appl. Eng. Agric., 2013; 29: 675-682.

[14] Qin W C, Qiu B J, Xue X Y, Chen C, Xu Z F, Zhou Q Q. Droplet deposition and control effect of insecticides sprayed with an unmanned aerial vehicle against plant hoppers. Crop Prot., 2016; 85: 79-88.

[15] Fritz B K, Hoffmann W C, Lan Y B. Evaluation of the EPA drift reduction technology (DRT) low-speed wind tunnel protocol. J. ASTM Int., 2009; 6: 102-129.

[16] Hoffmann W C, Fritz B K, Lan Y B. Evaluation of a proposed drift reduction technology high-speed wind tunnel testing protocol. J. ASTM Int., 2009; 6: 102-122

[17] Hilz E, Vermeer A W P. Spray drift review: the extent to which a formulation can contribute to spray drift reduction. Crop Prot., 2013; 44: 75-83.

[18] Reed W H. An analytical study of the effect of airplane wake on the lateral dispersion of aerial sprays. Report 3032. Langley, VA, USA: National Advisory Committee for Aeronautics, 1953.

[19] Xue X Y, Lan Y B. Agricultural Aviation Applications in USA. Transactions of the CSAM, 2013; 44(5): 194-201. (in Chinese)

[20] Teske M E, Bilanin A J, Barry J W. Decay of aircraft vortices near the ground. AIAA Journal, 1993; 31(8): 1531-1533.

[21] Teske M E, Bird S L, Esterly D M, Curbishley T B, Ray S L, Perry S G. AgDrift ${ }^{\circledR}$ : A model for estimating near-field spray drift from aerial applications. Environ. Toxicol. Chem., 2002; 21(3): 659-671.

[22] Teske M E, Thistle H W, Schou W C, Miller P C, Strager J M, Richardson B, et al. A review of computer models for pesticide deposition prediction. Transactions of the ASABE, 2011; 54(3): 789-801.

[23] Dumbauld R K, Bjorklund J R, Saterlie S F. Computer models for predicting aircraft spray dispersion and deposition above and within forest canopies: User's manual for the FSCBG computer program. Report 80e11. Davis, CA: U.S. Department of Agriculture Forest Service, 1980.

[24] Teske M E, Thistle H W. Aerial application model 
extension into the far field. ASAE Annual Meeting, 2003.

[25] Reed W H. An analytical study of the effect of airplane wake on the lateral dispersion of aerial sprays. Report 3032. Langley, VA, USA: National Advisory Committee for Aeronautics, 1953.

[26] Craig I P. The GDS model-a rapid computational technique for the calculation of aircraft spray drift buffer distances. Comput. Electron. Agr., 2004; 43(3): 235-250.

[27] Bartzanas M T, Zhu H. Karmakar S, Tamimi E, Katsoulas $\mathrm{N}$, et al. Computational fluid dynamics applications to improve crop production systems. Comput. Electron. Agr., 2013; 93: 151-167.

[28] Brown R B, Sidahmed M M. Simulation of spray dispersal and deposition from a forestry airblast sprayer. Part II: droplet trajectory model. Trans. ASAE, 2001; 44(1): 11-17.

[29] Nuyttens D, De Schampheleire M. Baetens K, Brusselman E, Dekeyser D, Verboven P. Drift from field crop sprayers using an integrated approach: results of a five-year study. Transactions of the ASABE, 2011; 54(2): 403-408.

[30] Molari G, Benini L, Ade G. Design of a recycling tunnel sprayer using CFD simulations. Trans. ASAE, 2005; 48(2): 463-468.

[31] Tsay J, Ozkan H E, Brazee, R D, Fox R D. CFD simulation of moving spray shields. Trans. ASAE, 2002; 45(1): 21-26.

[32] Reichard D L, Zhu H, Fox R D, Brazee R D. Computer simulation of variables that influence spray drift. Trans. ASAE, 1992; 35(5): 1401-1407.
[33] Zhu H, Reichard D L, Fox R D, Brazee R D, Ozkan H E. Simulation of drift of discrete sizes of water droplets from field sprayers. Trans. ASAE, 1994; 37(5): 1401-1407.

[34] Zhu H, Richard D L, Fox R D, Brazee R D, Ozkan H E. Collection efficiency of spray droplets on vertical targets. Trans. ASAE, 1996; 39(2): 415-422.

[35] Ryan S D, Gerber A G., Holloway A. A computational study on spray dispersal in the wake of an aircraft. Transactions of the ASABE, 2013; 56(3): 847-868.

[36] Zhang S C, Xue X Y, Qin W C, Sun Z, Ding S M, Zhou L X. Simulation and experimental verification of aerial spraying drift on N-3 unmanned spraying helicopter. Trans. CSAE, 2015; 31(3): 87-93. (in Chinese)

[37] Alderete T S. Simulator Aero Model Implementation. NASA Ames Research Center, Moffett Field, California, 1995.

[38] ETKIN. B. Dynamics of Flight. New York: John Wiley and Sons, INC, 1959.

[39] Roberts T W, Murman E M. Solution method for a hovering helicopter roter using the Euler equations. AIAA 85-0436, 1985

[40] Pomin H, Wagner S. Navier-Stokes analysis of helicopter rotor aerodynamics in hover and forward flight. AIAA 2001-0998, 2001.

[41] Orszag S A, Yakhot V, Flannery W S. Renormalization group modeling and turbulence, in international conference on near wall turbulence flows. 1993. 